\title{
Association of GSTM1 and GSTT1 polymorphism with lipid peroxidation in benign prostate hyperplasia and prostate cancer: A pilot study
}

\author{
Vivek Kumar ${ }^{\mathrm{a}}$, Chandra Shekhar Yadav ${ }^{\mathrm{a}}$, Sudip Kumar Datta ${ }^{\mathrm{a}}$, Satyender Singh ${ }^{\mathrm{c}}$, Rafat S Ahmed ${ }^{\mathrm{a}}$, \\ Sanjay Goel ${ }^{\mathrm{b}}$, Sanjay Gupta ${ }^{\mathrm{b}}$, Md. Mustafa ${ }^{\mathrm{a}}$, Rajesh Kumar Grover ${ }^{\mathrm{d}}$ and Basu Dev Banerjee ${ }^{\mathrm{a}, *}$ \\ ${ }^{a}$ Environmental Biochemistry and Molecular Biology Laboratory, Department of Biochemistry, University College \\ of Medical Sciences and GTB Hospital, University of Delhi, Dilshad Garden, Delhi, India \\ ${ }^{\mathrm{b}}$ Department of Surgery, University College of Medical Sciences and GTB Hospital, University of Delhi, Dilshad \\ Garden, Delhi, India \\ ${ }^{\mathrm{c}}$ Division of Biochemistry and Biotechnology, National Centre for Communicable Diseases, 22, Sham Nath Marg, \\ Delhi, India \\ ${ }^{\mathrm{d}}$ Director and CEO, Delhi State Cancer Institute, Dilshad Garden, Delhi, India
}

\begin{abstract}
Association of glutathione S-transferase (GST) $M 1$ and $T 1$ deletions with benign prostate hyperplasia (BPH) and prostate cancer is well reported. These enzymes metabolize numerous toxins thus protecting from oxidative injury. Oxidative stress has been associated with development of BPH and prostate cancer. The present study was designed to analyze role of GST deletions in development of oxidative stress in these subjects. GSTs are responsible for metabolism of toxins present in tobacco therefore effect of tobacco usage in study groups was also studied. Three groups of subjects: BPH (57 patients), prostate cancer (53 patients) and controls (46 subjects) were recruited. Genotyping was done using a multiplex polymerase chain reaction (PCR) method. Malondialdehyde (MDA) levels as marker of oxidative stress were estimated by measuring thiobarbituric acid reactive substance (TBARS) in plasma. Based on genotyping, subjects were categorized into: GSTM1+/GSTT1+, GSTM1-/GSTT1+, GSTM1+/GSTT1- and GSTM1-/GSTT1-. Significantly higher plasma MDA levels were noticed in GSTM1-/GSTT1- as compared to GSTM1+/GSTT1+ in all study groups. Double deletion (GSTM1-/GSTT1-) is associated with higher oxidative stress which might play a role in the pathogenesis of BPH and prostate cancer. However, other markers of oxidative stress should be analyzed before any firm conclusion.
\end{abstract}

Keywords: Glutathione S-transferase, malondialdehyde, genotypes, deletion

\section{Introduction}

Benign prostate hyperplasia $(\mathrm{BPH})$ is a non-malignant enlargement of the prostate leading to obstructive lower urinary tract symptoms (LUTs) affecting

*Corresponding author: Dr. B. D. Banerjee, M.Phil, PhD, Professor and Head (Medical Biochemistry, Faculty of Medical Sciences, DU). Department of Biochemistry, University College of Medical Sciences and Guru Tegh Bahadur Hospital, University of Delhi, Dilshad Garden, Delhi-110 095, India. Tel.: +91 11 22135362; Fax: +91 11 22590495; E-mail: banerjeebd@hotmail.com. more than $50 \%$ of men by the age 60 years and $90 \%$ by 85 years [1]. On the other hand, prostate cancer is a common malignancy in males and a leading cause of cancer-related mortality [2]. Reactive oxygen species (ROS) have been implicated in the etiology of both BPH and prostate cancer [3-5]. Oxidative stress results from either increased free radical generation and/or decreased antioxidant levels in the target cells/tissues/organs and is associated with most cancers $[6,7]$. Oxidative stress results in damage of critical cellular macromolecules including DNA, resulting in altered cellular function, a crucial event in carcinogen- 
esis [3]. Apart from base excision repair, which can repair oxidative DNA damage several mechanisms exist in humans to counteract the adverse effects of ROS [8]. Glutathione S-transferases (GSTs, EC 2.5.1.18) are a family of phase II xenobiotic metabolizing enzymes, protecting the body from oxidative insults by conjugating glutathione with various electrophilic compounds generated after activation by phase I enzymes [9]. Deletion of GSTM1 and GSTT1 genes, which are important isoenzymes of GST, leads to complete lack of activity of their enzymes. Absence of these enzymes due to homozygous deletions are implicated in poor elimination of carcinogenic substances including constituents of tobacco which are potential sources of ROS in our body, making individuals with these deletions susceptible to oxidative injury [10]. Studies have reported positive association of GSTM1 or GSTT1 polymorphism with increased risk of BPH and prostate cancer [1115]. Since these individual deletions are reported to be associated with increased risk, effect of deletion of both these genes could be additive. Recent studies from our laboratory reported association of deletion of both GSTM1 and GSTT1 with markers of oxidative stress like malondialdehyde (MDA) in disorders like diabetic nephropathy, non-diabetic nephropathy and adverse reproductive outcomes [16-18].

The association between smoking and prostate cancer is not unequivocal and some studies report increased risk only in more aggressive forms of the disease [19, 20]. Available literature suggests that smoking contributes to development of oxidative injury, reflected by higher lipid peroxidation products [21]. Moreover, association between smoking and GST genotypes has also been reported [10,19,21-23]. One study reported the association of GSTT1 polymorphism with increase in markers of lipid peroxidation and inflammation in smokers with relation to cardiovascular diseases [24].

Studies have also analyzed MDA as marker of oxidative stress and found significantly higher MDA levels in prostate cancer in both blood and prostatic tissue [4, 5]. However, role of GSTM1 and/or GSTT1 deletion in the development of MDA in prostate cancer or BPH has not been explored. Effect of tobacco usage (in India, tobacco chewing in form of gutka, pan-masala etc. is as common as smoking [25]) in these patients with GSTM1 and GSTT1 deletions is not well-elucidated. Hence, the main objective of the present study was to evaluate the role of concomitant deletion of GST and their interaction with tobacco usage in the possible development of oxidative stress in BPH and prostate cancer patients.

\section{Material and methods}

\subsection{Subjects}

A total of one hundred and fifty six subjects were included in the study, fifty seven newly diagnosed prostate cancer patients, fifty three newly diagnosed BPH patients and forty six controls. All study subjects were North Indians who are ethnically known to be Caucasoid Aryans [26]. All the groups were matched regarding age; matching was done within 3 year-intervals within each group. Information regarding age, tobacco usage associated medical history etc. was obtained as per pre-designed questionnaire. Persons using tobacco (any form cigarette, gutka, panmasala etc.) for a minimum period of one year at or within a year of diagnosis (BPH and prostate cancer)/inclusion in study (controls) were included as current tobacco users, whereas, previous tobacco users were defined as those who quit tobacco usage at least a year before diagnosis/inclusion [27]. Subjects with history of chronic liver dysfunction, diabetes mellitus were excluded because of their suspected role in pathogenesis of both BPH and prostate cancer $[1,28]$. Also subjects with history of any other chronic disease were excluded. All subjects were either recruited from the Department of Surgery, Guru Teg Bahadur Hospital, Delhi and Delhi State Cancer Institute, Delhi. Necessary ethical clearances were approved by the Institutional Ethical Committee for Human Research. Written informed consent was obtained from each subject prior to their inclusion in the study. Study groups (BPH, prostate cancer and controls) were defined on the basis of clinical, biochemical, radiological as well as histopathological examination of tissue biopsy samples. Prostate cancer subjects were histologically confirmed cases. Subjects with LUTs, plasma prostate specific antigen (PSA) levels $>4 \mathrm{ng} / \mathrm{ml}$ and prostate volume $>20 \mathrm{cc}$ were included as BPH. Controls were free from any chronic disease on the basis of thorough medical examination including chest X-rays, urine and blood tests for various tumor markers, gastric endoscopy, abdominal ultrasound, colon enema. Controls were defined as persons with no LUTs, plasma PSA levels $<4 \mathrm{ng} / \mathrm{ml}$ and prostate volume $<20 \mathrm{cc}$. Some of the eligible controls were selected from our previous studies [29-31]. About five $\mathrm{ml}$ of peripheral venous blood was collected from each subject in Na-EDTA vaccutainers. Plasma was separated by centrifugation and refrigerated at $-20^{\circ} \mathrm{C}$ until analysis. 
Table 1

General characteristics of study groups

\begin{tabular}{|c|c|c|c|}
\hline & $\mathrm{PC}(n=57)$ & $\mathrm{BPH}(n=53)$ & Controls $(n=46)$ \\
\hline $\begin{array}{l}\text { Mean age } \pm \text { SD } \\
\text { (Range) }\end{array}$ & $\begin{array}{c}69.2 \pm 9.1 \\
(42-86)\end{array}$ & $\begin{array}{c}66.1 \pm 9.3 \\
(45-81)\end{array}$ & $\begin{array}{c}68.8 \pm 5.9 \\
(56-82)\end{array}$ \\
\hline \multicolumn{4}{|l|}{ Age categories (\%) } \\
\hline$>50$ & $1(1.6)$ & $2(3.8)$ & $0(0)$ \\
\hline $50-59$ & $10(17.6)$ & $11(20.7)$ & $10(21.8)$ \\
\hline $60-69$ & $19(33.4)$ & $18(34.0)$ & $13(28.2)$ \\
\hline$<70$ & $27(47.4)$ & $22(41.5)$ & $23(50.0)$ \\
\hline \multicolumn{4}{|l|}{ Tobacco usage (\%) } \\
\hline Current & $18(31.6)$ & $13(24.5)$ & $9(19.6)$ \\
\hline Previous & $10(17.5)$ & $9(17.0)$ & $12(26.1)$ \\
\hline Never & $29(50.9)$ & $31(58.5)$ & $25(54.3)$ \\
\hline \multicolumn{4}{|c|}{ Genotype (combined) (\%) } \\
\hline GSTM1+/ GSTT1+ (\%) & $10(17.5)$ & $12(22.6)$ & $17(37.0)$ \\
\hline GSTM1-/ GSTT1+ (\%) & $18(31.6)$ & $9(17.0)$ & $7(15.2)$ \\
\hline GSTM1+/ GSTT1- (\%) & $13(22.8)$ & $20(37.7)$ & $14(30.4)$ \\
\hline GSTM1-/ GSTT1- (\%) & $16(28.1)$ & $12(22.7)$ & $8(17.4)$ \\
\hline \multicolumn{4}{|c|}{ Genotype (individual) (\%) } \\
\hline GSTM1+ $(\%)$ & $23(40.4)$ & $32(61.1)$ & $31(67.4)$ \\
\hline GSTM1- (\%) & $34(59.6)$ & $21(38.9)$ & $15(32.6)$ \\
\hline GSTT1+ (\%) & $28(49.1)$ & $21(40.7)$ & $24(52.2)$ \\
\hline GSTT1- (\%) & $29(50.9)$ & $32(59.3)$ & $22(47.8)$ \\
\hline
\end{tabular}

\subsection{GSTM1 and GSTT1 genotyping}

DNA was extracted from whole blood using DNA extraction kit (Omniprep $^{\mathrm{TM}}$, GBiosciences, USA). A single assay using multiplex polymerase chain reaction (PCR) was performed for simultaneous gene amplification [32] using the Eppendorf Mastercycler Gradient5331 thermocycler. Briefly, 100 ng of DNA was amplified in $50 \mu \mathrm{l}$ multiplex reaction mixture containing $30 \mathrm{pM}$ of each of the following GSTM1 primers (MF5' GAA CTC CCT GAA AAG CTA AAG C 3' and MR - 5' GTT GGG CTC AAA TAT ACG GTG G 3') and of the following GSTT1 primers (TF - 5' TTC CTT ACT GGT CCT CAC ATC TC 3' and TR- 5' TCA CCG GAT CAT GGC CAG CA 3'). As an internal control, the exon 7 of the $C Y P 1 A 1$ gene fragment was also co-amplified (CF- 5' GAA CTG CCA CTT CAG CTG TCT 3' and CR- 5' CAG CTG CAT TTG GAA GTG CTC 3') in a mastermix consisting of $1.5 \mathrm{mM} \mathrm{MgCl}_{2}$, $200 \mu \mathrm{M}$ dNTPs (Bangalore Genei, Banglore, India), $5 \mu \mathrm{l}$ 10X PCR buffer (500 mM KCl, $100 \mathrm{mM}$ Tris-HCl, $\mathrm{pH}$ 9.0) and $2 \mathrm{U}$ Taq DNA polymerase (New England Bio Labs, Beverley, MA). The PCR thermocyclic condition included an initial denaturation at $94^{\circ} \mathrm{C}$ for 5 min followed by 35 cycles of $2 \mathrm{~min}$ at $94^{\circ} \mathrm{C}, 1 \mathrm{~min}$ at $59^{\circ} \mathrm{C}$ and $1 \mathrm{~min}$ at $72^{\circ} \mathrm{C}$ followed by a final extension of $10 \mathrm{~min}$ at $72^{\circ} \mathrm{C}$. The final PCR product from coamplification of GSTM1 (215 bp), GSTT1 (480 bp) and CYP1A1 (312 bp) was visualized after electrophoresis in ethidium bromide-stained $2 \%$ agarose gel.

\subsection{Malondialdehyde levels}

Lipid peroxidation, a known marker of oxidative stress was determined by spectrophotometric measurement of malondialdehyde (MDA), a thiobarbituric acid reactive substance (TBARS) in plasma [33]. MDA levels were expressed in $\mathrm{nmol} / \mathrm{ml}$.

\subsection{Statistical analysis}

Statistical comparison was performed using SPSS software package (version 17.0 for Windows; SPSS, Chicago, IL) at Department of Biostatistics and Medical Information, UCMS and GTB Hospital. One-way ANOVA with Tukey's multiple comparisons as posthoc test was used for analysis of differences in mean MDA levels in four identified genotypes in each study group.

The distribution of population characteristics for study groups were compared by Fischer exact test and student t-test. Chi-square $\left(\chi^{2}\right)$ test was used to compare deletion of individual genes, $\mathrm{p}$-value $<0.05$ was considered as significant.

\section{Results}

General characteristics of the different study groups like age, tobacco usage and genotype distribution are presented in Table 1. Age was not significantly differ- 
Table 2

Anthropometric and other characteristics of study groups

\begin{tabular}{lccc}
\hline & Control $(n=46)$ & $\mathrm{BPH}(n=53)$ & Prostate cancer $(n=57)$ \\
\hline PSA levels (mean $\pm \mathrm{SD})$ & $2.4 \pm 0.76$ & $21.9 \pm 13.5^{*}$ & $33.2 \pm 21.7^{*}$ \\
Marital status & & & \\
Yes & $44(95.7)$ & $52(98.1)$ & $55(96.5)$ \\
No & $2(4.3)$ & $1(1.9)$ & $2(3.5)$ \\
$\quad$ History of cancer in first degree relatives & $4(8.6)$ & $9(17.0)$ & $13(22.8)^{*}$ \\
Yes & $42(91.4)$ & $44(83.0)$ & $44(77.2)$ \\
No & $26(56.5)$ & $29(54.7)$ & $28(49.1)$ \\
Education & $20(43.5)$ & $24(45.3)$ & $29(50.9)$ \\
Secondary or less & $28(60.9)$ & $30(56.6)$ & $31(54.4)$ \\
Post Secondary & $18(39.1)$ & $23(43.4)$ & $26(45.6)$ \\
$\begin{array}{l}\text { Physical activity } \\
\text { Low/moderate }\end{array}$ & & & \\
$\begin{array}{l}\text { Strenuous/very strenuous } \\
\text { Body mass index }\end{array}$ & $37(80.4)$ & $45(84.9)$ & $45(78.9)$ \\
$\begin{array}{l}\text { Underwieght/normal } \\
\text { Overwieght/ obese } \\
\text { Income }\end{array}$ & $9(19.6)$ & $8(15.1)$ & $12(21.1)$ \\
Low/upper low & & & \\
Low middle/upper middle/upper & $41(89.1)$ & $44(83.0)$ & $49(86.0)$ \\
\hline
\end{tabular}

*significant at $p<0.05$;

${ }^{\delta}$ Classification of income is based of format prescribed for assessing socioeconomic status in Indian population. Low $(<1 \$$ /day or ₹ 1500 per month), upper low ( $\$$ /day or ₹ 1500 per month to $<₹ 3000$ per month), low middle (3000 to $<₹ 9000$ per month), upper middle ( $\geqslant 9000$ to $<₹ 15000$ per month), upper ( $\geqslant ₹ 15000$ per month) [41].

Table 3

MDA levels in genotypic subgroups in study groups

\begin{tabular}{lcccc}
\hline Genotypes & GSTM1+/ GSTT1+ & GSTM1-/ GSTT1+ & GSTM1+/ GSTT1- & GSTM1-/ GSTT1- \\
\hline Prostate cancer & $1.96 \pm 0.98$ & $2.28 \pm 0.85$ & $2.11 \pm 1.02$ & $4.84 \pm 1.59$ \\
BPH & $1.91 \pm 0.68$ & $4.27 \pm 1.41$ & $2.16 \pm 1.17$ & $4.30 \pm 1.21$ \\
Controls & $2.16 \pm 0.90$ & $2.97 \pm 1.28$ & $2.91 \pm 1.26$ & $4.31 \pm 1.17$ \\
\hline
\end{tabular}

ent amongst the study groups. GSTMI deletion was significantly higher in prostate cancer subjects as compared to controls $\left(\chi^{2}=6.4, p\right.$-value $\left.=0.01\right)$. However, no association was observed in BPH with either GSTM1 or GSTT1 deletion.

Anthropometric and other characteristics of study groups like PSA (mean $\pm \mathrm{SD}$ ), marital status, family history of prostate cancer, education, physical activity, body mass index (BMI) and income are presented in Table 2. PSA levels (mean $\pm \mathrm{SD}$ ) were significantly higher in both BPH and prostate cancer groups as compared to controls ( $p$-value $=0.0001$ and 0.005 respectively), Also significantly higher frequency of cases with history of cancer in first degree relatives as compared to the controls was observed $(22.8 \%$ vs $8.6 \%, p$-value $=$ 0.02 ).

Following genotyping, four genotypes were identified amongst our subjects: GSTMI+/GSTT1+ (nodeletion), GSTM1-/GSTT1+ (only GSTM1- deletion), GSTM1+/GSTTI - (only GSTTI-deletion) and GSTMI/GSTT1- (both-deletion). MDA levels in these genotypic subgroups in prostate cancer, BPH and con- trols are presented in Table 3. To study the effect of genotypic variation on possible development of oxidative stress, MDA levels in plasma were estimated and analyzed on the basis of above mentioned genotypic sub-groups as shown in Fig. 1. Mean MDA levels in prostate cancer, BPH and controls were not found to be significantly different from each other (Fig. 1). In control subjects, MDA levels were significantly higher in GSTM1-/GSTT1-group compared to $G S T M 1+/ G S T T 1+$ genotype. However, in BPH patients MDA levels were higher amongst GSTMI/GSTT1- and GSTM1-/GSTT1+ groups in comparison to other two genotypes (GSTM1+/GSTTI- and $G S T M 1+/ G S T T 1+)$ whereas, in prostate cancer patients MDA levels were again significantly higher amongst GSTM1-/GSTT1 - cases in comparison to other three genotypes (GSTM1+/GSTT1-, GSTM1-/GSTT1+ and $G S T M 1+/ G S T T 1+$ ). Significant difference in mean MDA levels were not observed due to single gene deletion in any group (data not presented).

We analyzed the association between tobacco usage and MDA levels in all study groups. MDA levels were 


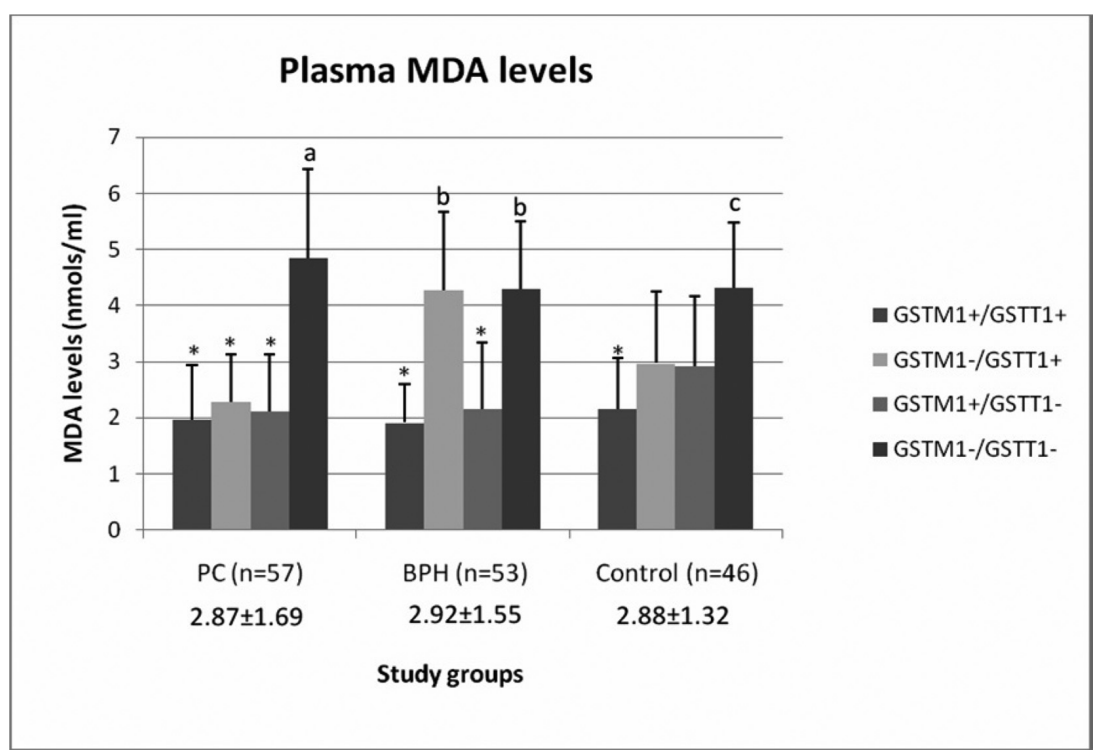

Fig. 1. Comparison of MDA levels in different genotypes within each study group. PC: Prostate cancer, BPH: Benign prostate hyperplasia; a: significantly different from (*) GSTM1+/GSTT1+, GSTM1-/GSTT1+ and GSTM1+/GSTT1- genotypes at $p<0.05$ within prostate cancer group. b: significantly different from (*) GSTM1+/GSTT1+ and GSTM1+/GSTT1- genotypes at $p<0.05$ within BPH group. c: significantly different from $(*)$ GSTM1+/GSTT1+ genotypes at $p<0.05$ within control group.

compared amongst subjects with one/more deletions with subjects with no deletions in all subject groups amongst current tobacco users, previous tobacco users and non-users. However, statistically significant differences were not observed (data not presented).

\section{Discussion}

GST enzymes catalyze the conjugation of electrophilic compounds to glutathione and play an important role in the protection of DNA and other macromolecules from oxidative damage. Amongst the principal isoenzymes of GST, GSTM1 is involved in the metabolism of a possible carcinogen, styrene oxide (an intermediate of styrene synthesis) [34]. It also catalyses metabolism of carcinogenic benzo $[\alpha]$ pyrene7,8-diol9.10-oxide, found in tobacco smoke [35]. On the other hand, GSTT1 enzyme is reported to be involved in metabolism of potentially carcinogenic dichloromethane and ethylene oxide [36]. GSTP1 is reported to be involved in the metabolism of toxic compounds like acrolien, a compound found in tobacco and associated with lung cancer $[37,38]$. Since GSTM1 and GSTT1 genes are reported to be deleted causing a loss in enzymatic activity of these isoenzymes deletion of both genes concomitantly might lead to more susceptibility for various cancers including that of prostate.
Hence, not surprisingly, GST polymorphisms are reported to be associated with higher risk of cancers including that of prostate [9-12,21]. However, the exact mechanism by which GST polymorphisms might lead to higher cancer risk is not well understood. Studies also report risk of BPH due to GST polymorphism [15]. Hence, the present pilot study was planned to investigate the association of concomitant deletions of GST genes with a marker of oxidative stress in subjects with $\mathrm{BPH}$ or prostate cancer.

We studied MDA levels one of the most established markers of oxidative stress and compared the levels amongst different genotypic subgroups within each study group. Significantly higher MDA levels observed amongst GSTM1-/GSTT1- compared to $G S T M 1+/ G S T T 1+$ in all subject groups indicate higher oxidative stress in individuals with double deletions. These observations indicate a possible role of concomitant deletion of GST in oxidative stress development in these subjects. In the present work MDA was assessed in blood to reflect association of oxidative stress with $\mathrm{BPH}$ and prostate cancer as prostatic tissue is not always easy to obtain for analysis [4]. However, evidence suggests that blood levels provide a reliable indication about the status of different stress markers including MDA in different human tissues [39]. Literature is scarce regarding association of MDA levels with GST gene deletion in BPH and prostate cancer patients. 
Furthermore, only one study has reported association of both GSTM1 and GSTT1 deletion with increased risk of oral cancer [40]. Recently, similar studies from our laboratory have associated deletion of these genes with different markers of oxidative stress in diabetic/nondiabetic nephropathy and preterm labour [16-18]. Our results indicate a possible interaction between oxidative stress, concomitant deletion of GST and prostate cancer. However, further studies are required to analyze and validate these observations.

Previously, numerous studies have analyzed the role of GSTs polymorphism with risk of prostate cancer. We have found significantly higher frequency of GSTM1 deletion in prostate cancer patients compared to controls. This observation is in agreement with several previous studies reporting significant association of GSTM1 deletion with increased risk of prostate cancer [11]. However, some other studies did not find any association of GSTM1 deletion with prostate cancer [12].

Unlike the prostate cancer patients, significantly higher MDA levels was observed in BPH subjects amongst GSTM1-/GSTT1+ groups compared to GSTM1+/GSTT1+ genotypes (Fig. 1). Hayek et al. (2006) reported GSTT1+ genotype to be associated with elevated markers of lipid peroxidation and inflammation in patients with diabetes mellitus [24]. Besides, one recent study reported GSTM1 null genotype to be associated with increased risk of BPH [15]. Together, these observations suggest that presence of GSTT1 and/or absence of GSTM1 genotype might have significance in the possible development of oxidative stress in $\mathrm{BPH}$ patients and hence in the underlying pathogenesis.

Interaction of tobacco usage with GST polymorphism has been reported to increase the risk of prostate cancer [21], however no significant relation of GST polymorphisms was observed with tobacco usage in the study groups. The results of our pilot study indicate that concomitant deletion (GSTM1- and GSTT1-) is associated higher plasma MDA levels, a marker of oxidative stress in both BPH and prostate cancer subjects. Hence, it may be concluded that double deletion might be a risk factor for BPH and prostate cancer. However, oxidative stress levels might also be modified by various other factors like chronic inflammation, diet supplementations etc. The fact that this is a pilot-study, based on cross-sectional design with small sample size in some sub-groups may limit our conclusion towards postulating the population-attributable risk. Hence, these results should be validated with various other markers of oxidative stress in poly-ethnic prospective studies with larger sample size using more sensitive mRNA expression analysis tools etc. Further work in this area will elucidate the role of these multi-functional antioxidant enzymes, which might be helpful in defining population with higher risk and designing meaningful therapeutic and preventive interventions.

\section{Acknowledgements}

One of the authors Vivek Kumar is thankful to University Grant Commission for providing Junior Research Fellowship (NET) and funding for the research material from Ministry of Environment and Forest (Govt. of India).

\section{References}

[1] J.K. Parsons, H.B. Carter, A.W. Partin, G.B. Windham, E.J. Metter, L. Ferrucci, P. Landis and E.A. Platz, Metabolic factors associated with benign prostatic hyperplasia, J Clin Endocr Metab 91 (2006), 2562-2568.

[2] A. Jemal, R. Siegel, E. Ward, Y. Hao, J. Xu and M.J. Thun, Cancer statistics, 2009, Ca-Cancer J Clin 59 (2009), 225-249.

[3] J.E. Klaunig, Y. Xu, J.S. lsenberg, S. Bachowski, K.L. Kolaja, J. Jiang, D.E. Stevenson and E.F. Walborg, Jr., The role of oxidative stress in chemical carcinogenesis, Environ Health Persp 106 (1998), 289-295.

[4] A. Aydin, Z. Arsova-Sarafinovska, A. Sayal, A. Eken, O. Erdem, K. Erten, Y. Özgök and A. Dimovski, Oxidative stress and antioxidant status in non-metastatic prostate cancer and benign prostatic hyperplasia, Clin Biochem 39 (2006), 176179.

[5] H. Biri, H.S. Ozturk, M. Kacmaz, K. Karaca, H. Tokucoglu and I. Durak, Activities of DNA turnover and free radical metabolizing enzymes in human prostate tissue, Cancer Invest 17 (1999), 314-319.

[6] M.A. Trush and T.W. Kensler, An overview of the relationship between Oxidative stress and chemical carcinogenesis, Free Radic Bio Med 10 (1991), 201-209.

[7] C. Rice-Evans and R. Burdon, Free radical-lipid interactions and their pathological consequences, Prog Lipid Res 32 (1993), 71-110.

[8] E. Seeberg, L. Eide and M. Bjørås, The base excision repair pathway, Trends Biochem Sci 20 (1995), 391-397.

[9] C. Ntais, A. Polycarpou and J.P.A. Ioannidis, Association of GSTM1, GSTT1, and GSTP1 gene polymorphisms with the risk of prostate cancer: a meta-analysis, Cancer Epidemiol Biomark Prev 14 (2005), 176-181.

[10] T.R. Rebbeck, Molecular epidemiology of the human glutathione $S$-transferase genotypes GSTM1 and GSTT1 in cancer susceptibility, Cancer Epidemiol Biomark Prev 6 (1997), 733-743.

[11] I. Agalliu, W.J. Langeberg, J.W. Lampe, C.A. Salinas and J.L. Stanford, Glutathione Stransferase M1, T1, and P1 polymorphisms and prostate cancer risk in middle-aged men, Prostate 66 (2006), 146-156. 
[12] T.R. Rebbeck, A.H. Walker, J.M. Jaffe, D.L. White, A.J. Wein and B.S. Malkowicz, Glutathione $S$-Transferase- $\mu$ (GSTMI) and $-\theta$ (GSTT1) genotypes in the etiology of prostate cancer, Cancer Epidemiol Biomark Prev 8 (1999), 283-287.

[13] D.S.L. Srivastava, A. Mandhani, B. Mittal and R.D. Mittal, Genetic polymorphism of glutathione S-transferase genes (GSTM1, GSTT1 and GSTP1) and susceptibility to prostate cancer in Northern India, BJU int 95 (2005), 170-173.

[14] S. Mallick, R.M. Romana, P. Blanchet and L. Multigner, GSTM1 and GSTT1 polymorphisms and the risk of prostate cancer in a Caribbean population of African descent, Urology 69 (2007), 1165-1169.

[15] R.D. Mittal, P. Kesarwani, R. Singh, D. Ahirwar and A. Mandani, GSTM1, GSTM3 and GSTT1 gene variants and risk of benign prostate hyperplasia in Northern India. Dis Markers 26 (2009), 85-91.

[16] S.K. Datta, V. Kumar, R.S. Ahmed, A.K. Tripathi, O.P. Kalra and B.D. Banerjee, Effect of GSTM1 and GSTT1 double deletion in development of oxidative stress in diabetic nephropathy patients, Indian J Biochem Bio 47 (2010), 100-103.

[17] M.D. Mustafa, R. Pathak, T. Ahmed, R.S. Ahmed, A.K. Tripathi, K. Guleria and B.D. Banerjee, Association of glutathione S-transferase M1 and T1 gene polymorphisms and oxidative stress markers in preterm labor, Clin Biochem $\mathbf{4 3}$ (2010), 1124-1128.

[18] S. K. Datta, V. Kumar, R. Pathak, A.K. Tripathi, R.S. Ahmed, O.P. Kalra and B.D. Banerjee, Association of glutathione $S$ transferase $M 1$ and $T 1$ gene polymorphism with oxidative stress in diabetic and non diabetic chronic kidney disease, Ren Fail 32 (2010), 1189-1195.

[19] S.N. Kelada, S.L.R. Kardia, A.H. Walker, A.J. Wein, B.S. Malkowicz and T.R. Rebbeck, The Glutathione S-Transferase$\mu$ and - $\theta$ Genotypes in the Etiology of Prostate Cancer: Genotype-Environment Interactions with Smoking, Cancer Epidemiol Biomark Prev 9 (2000), 1329-1334.

[20] K. Zu and E. Giovannucci, Smoking and aggressive prostate cancer: a review of the epidemiologic evidence, Cancer Cause Control 20 (2009), 1799-1810.

[21] J.D. Morrow, B. Frei, A.W. Longmire, J.M. Gaziano, S.M. Lynch, Y. Shyr, W.E. Strauss, J.A. Oates and L.J. Roberts, Increase in circulating products of lipid peroxidation (F2isoprostanes) in smokers, New Engl J Med 332 (1995), 1198 1203.

[22] K. Zu and E. Giovannucci, Smoking and aggressive prostate cancer: a review of the epidemiologic evidence, Cancer Cause Control 20 (2009), 1799-1810.

[23] I. Agalliu, W.J. Langeberg, J.W. Lampe, C.A. Salinas and J.L. Stanford, Glutathione Stransferase M1, T1, and P1 polymorphisms and prostate cancer risk in middle-aged men, Prostate 66 (2006), 146-156

[24] T. Hayek, J.W. Stephens, C.S. Hubbart, J. Acharya, M.J. Caslake, E. Hawe, G.J. Miller, S.J. Hurel and S.E. Humphries, A common variant in the glutathione $S$ transferase gene is associated with elevated markers of inflammation and lipid peroxidation in subjects with diabetes mellitus, Atherosclerosis 184 (2006), 404-412.

[25] M. Rani, S. Bonu, P. Jha, S.N. Nguyen and L. Jamjoum, Tobacco use in India: prevalence and predictors of smoking and chewing in a national cross sectional household survey, Tob Control 12 (2003), e4.
[26] J. Roland and L. Breton, Atlas of the Languages and Ethnic Communities of South Asia, Saga publication, New Delhi, 1993, pp. 21-39.

[27] N.L. Kobrinsky, M.G. Klug, P.J. Hokanson, D.E. Sjolander and L. Burd, Impact of smoking on cancer stage at diagnosis, J Clin Oncol 21 (2003), 907-913.

[28] A.W. Hsing, Y. Gao, S. Chua, Jr, J. Deng and F.Z. Stanczyk, Insulin resistance and prostate cancer risk, $J$ Natl Cancer I 95 (2003), 67-71.

[29] S. Singh, V. Kumar, S. Thakur, B.D. Banerjee, S.S. Grover, D.S. Rawat, S.T. Pasha, S.K. Jain, S. Lal and A. Rai, Genetic polymorphism of Glutathione S-Transferase M1 and T1 in Delhi population of North India, Environ Toxicol Phar 28 (2009), 25-29.

[30] V. Kumar, S. Singh, R.S. Ahmed, B.D. Banerjee, T. Ahmed and S.T. Pasha, Frequency of CYP1B1 polymorphic variation in North Indian population, Environ Toxicol Phar 28 (2009), 392-396.

[31] R. Gupta, V. Kumar, K. Luthra, B.D. Banerjee and M.S. Bhatia, Polymorphisms in Apolipoprotein E among migraineurs and tension type headache subjects, The J Headache Pain 10 (2009), 115-120.

[32] S.Z. Abdel-Rahman, R.A. El-Zein, W.A. Anwar and W.W. $\mathrm{Au}, \mathrm{A}$ multiplex PCR procedure for polymorphic analysis of GSTM1 and GSTT1 genes in population studies, Cancer Lett 107 (1996), 229-233.

[33] K. Satoh, Serum lipid peroxide in cerebrospinal disorder determined by a new colorimetric method, Clin Chim Acta $\mathbf{9 0}$ (1978), 37-43.

[34] M. Warholm, C. Guthenberg and B. Mannervik, Molecular and catalytic properties of glutathione transferase mu from human liver: an enzyme efficiently conjugating epoxides, Biochemistry 22 (1983), 3610-3617.

[35] B. Ketterer, Protective role of glutathione and glutathione transferases in mutagenesis and carcinogenesis, Mutat Res $\mathbf{2 0 2}$ (1988), 343-361.

[36] S. Pemble, K.R. Schroeder, S.R. Spencer, D.J. Meyer, E. Hallier, H.M. Bolt, B. Ketterer and J.B. Taylor, Human glutathione S-transferase Theta (GSTT1): cDNA cloning and the characterization of a genetic polymorphism, Biochem J 300 (1994), 271-276.

[37] K. Berhane and B. Mannervik, Inactivation of the genotoxic aldehyde acrolein by human glutathione transferases of classes alpha, mu, and pi, Mol Pharmacol 37 (1990), 251-254.

[38] Z. Feng, W. Hu, Y. Hu and M. Tang, Acrolein is a major cigarette-related lung cancer agent: Preferential binding at p53 mutational hotspots and inhibition of DNA repair, $P$ Natl Acad Sci USA 103 (2006), 15404-15409.

[39] A.S.Veskoukis, M.G. Nikolaidis, A. Kyparos and D. Kouretas, Blood reflects tissue oxidative stress depending on biomarker and tissue studied, Free Radic Bio Med 47 (2009), 1371-1374.

[40] S. Gronau, D. Koenig-Greger, M. Jerg and H. Riechelmann, GSTM1 enzyme concentration and enzyme activity in correlation to the genotype of detoxification enzymes in squamous cell carcinoma of oral cavity, Oral Dis 9 (2003), 62-67.

[41] A.B. Patel, A.S. Prabhu, M.J. Dibley and L.R. Kulkarni, A tool for rapid socioeconomic assessment, Indian J Pediat $\mathbf{7 4}$ (2007), 349-352. 


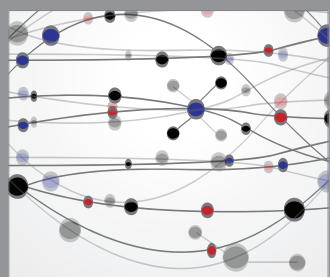

The Scientific World Journal
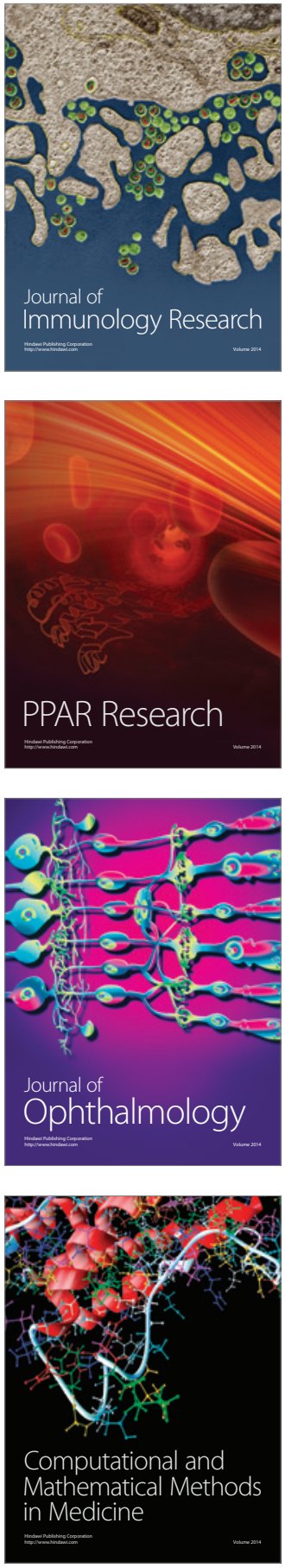

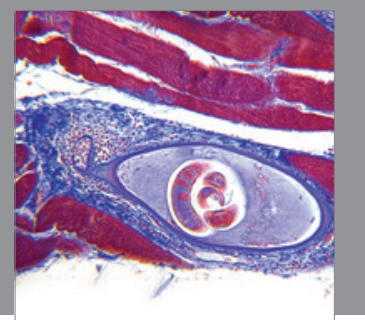

Gastroenterology

Research and Practice
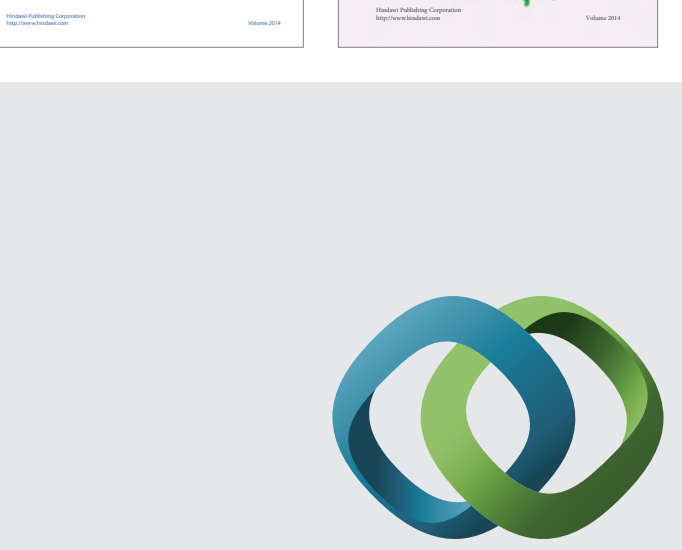

\section{Hindawi}

Submit your manuscripts at

http://www.hindawi.com
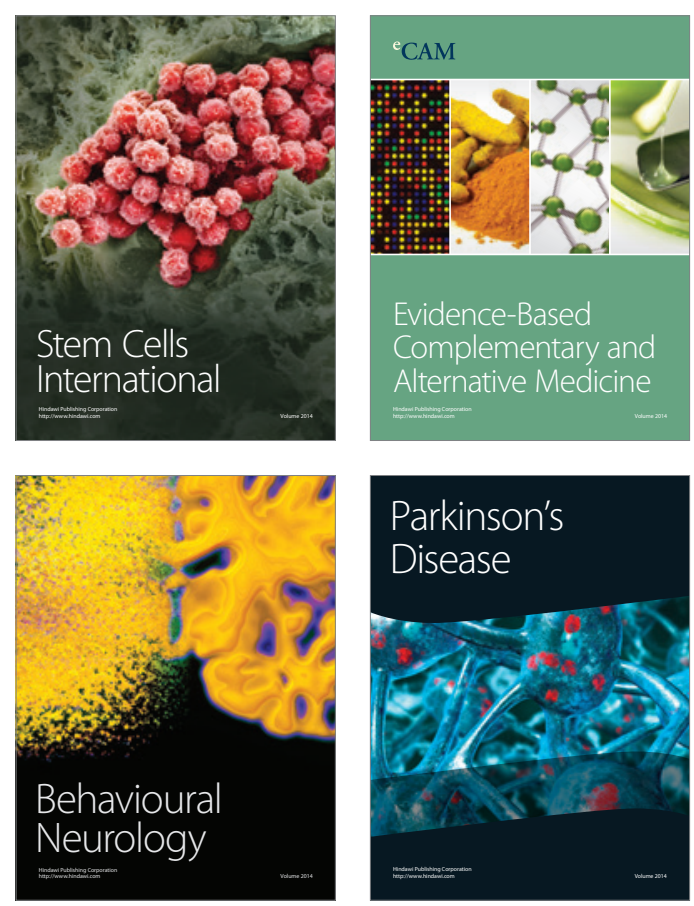

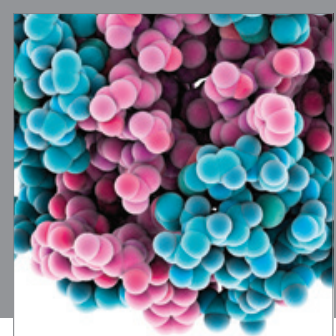

Journal of
Diabetes Research

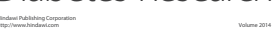

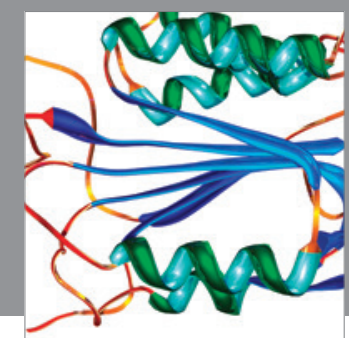

Disease Markers
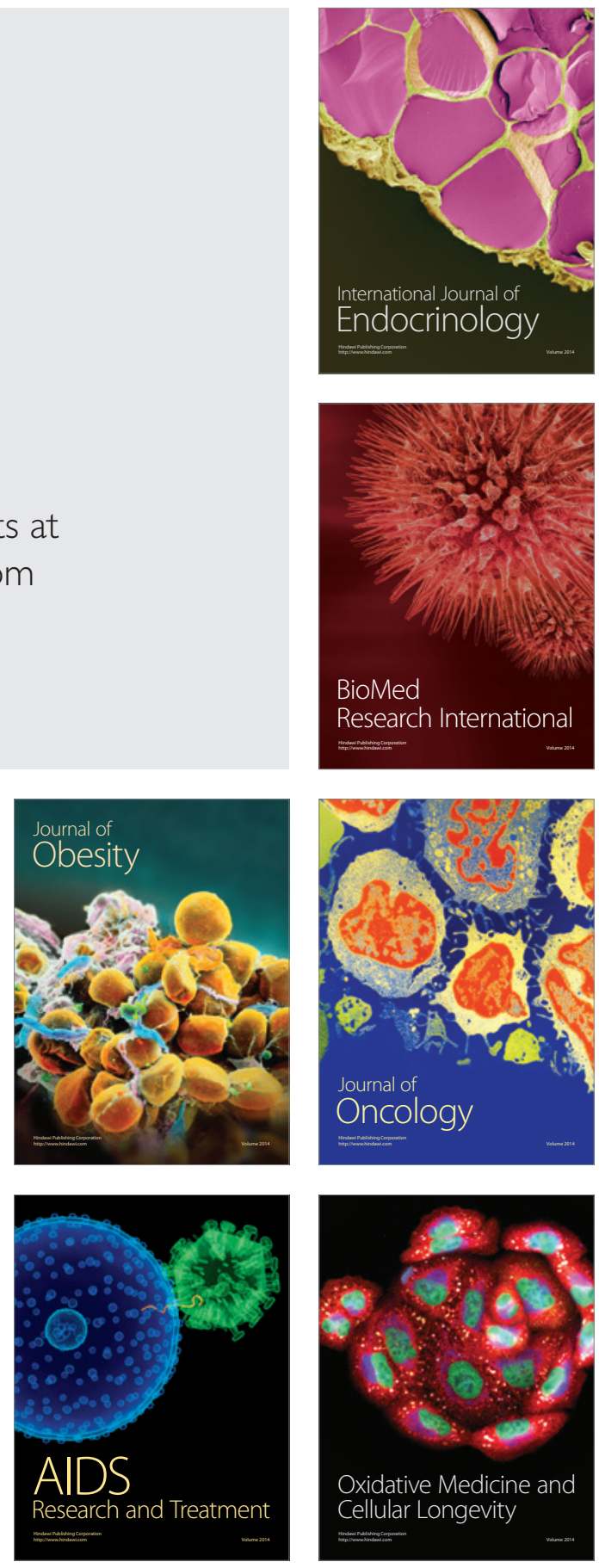